- The method used for assessing facial swelling in the present study has not been evaluated in the past. Although this is not a new method, the use of a point counting technique to measure the area drawn on postero-anterior skull radiographs in order to evaluate facial swelling is new.

- The use of a haemostatic agent in the extraction cavity had no significant effect on reducing the facial swelling after third molar surgery.

- This study encourages future studies which will investigate the relation between facial swelling and bleeding,

\title{
A prospective randomised cross-over study of the effect of local haemostasis after third molar surgery on facial swelling: an exploratory trial
}

\author{
A. Alkan, ${ }^{1}$ M. Metin, ${ }^{2}$ S. Arici ${ }^{3}$ and I. Sener ${ }^{4}$
}

Objective This study was undertaken in order to evaluate the effects of local haemostasis on the facial swelling induced in patients undergoing surgical removal of impacted mandibular third molar teeth.

Design A prospective randomised cross-over study.

Setting Department of Oral and Maxillofacial Surgery, Samsun, Turkey, 2002.

Materials and methods Twenty-five healthy patients who were having bilaterally symmetric, impacted third molar extraction were studied. The teeth were removed in two separate operations by two surgeons who had equal academic degrees. The patients were assigned to test and control groups by random selection. One side served as control and on the other local haemostasis was achieved by a haemostatic agent (oxidised regenerated cellulose). This was placed into the socket following gauze packing for 3 minutes (test). The operating time therefore significantly increased in the test group. Data was collected regarding the operating time, mouth opening and facial swelling.

Results There were no statistically significant differences between the test and control groups for the mean values of the facial swelling and mouth opening at day 1 and 3 post-operatively $(P>0.05)$. Operating time was statistically longer for the test group than the control $(P<0.001)$.

Conclusion This study has shown that establishment of local haemostasis after removal of impacted mandibular third molars is not so effective in preventing facial swelling.

\section{INTRODUCTION}

Impacted third molar surgery is the most common procedure performed by oral surgeons which often produces tissue trauma resulting in an inflammatory reaction and soft tissue swelling. By controlling the extent of the inflammatory process associated with the surgical procedure, these sequelae may also be reduced.

\footnotetext{
${ }^{1 *}, 2$ Associate Professor, ${ }^{4}$ Research Assistant, DDS; Department of Oral and Maxillofacial Surgery, Faculty of Dentistry, Ondokuz Mayis University, Samsun, Turkey; ${ }^{3}$ Associate Professor, DDS, PhD, Department of Orthodontics, Faculty of Dentistry, Ondokuz Mayis University, Samsun, Turkey

*Correspondence to: Dr Alper Alkan, Ondokuz Mayis Üniversitesi, Dis Hekimligi Fakültesi, 55139, Kurupelit, Samsun, Turkey

Email:alpera@omu.edu.tr
}

\section{Refereed paper}

Received 20.12.02; Accepted 03.06.03

doi:10.1038/sj.bdj.4811421

๑ British Dental Journal 2004; 197: 42-44
Several types of medications such as steroids, antihistamines and other anti-inflammatory drugs have been used to inhibit these sequelae. $^{2-4}$ Non-medication methods used to minimise facial swelling after third molar surgery could be followed as; secondary closure, primary closure and tube drain, secondary closure together with surgical drain, cold dressing, and soft laser application etc. ${ }^{1,5-8}$ None of the studies on third molar surgery related to facial swelling explore the effects of bleeding on facial oedema resulting from extraction trauma. The purpose of this study is to clarify this issue.

\section{MATERIALS AND METHODS}

Patients were screened clinically and radiographically. The criterion for participation in the study was the presence of bilaterally symmetric and impacted mandibular third molars requiring removal of alveolar bone. The reasons for extraction were resorption of adjacent teeth, disease of a follicle including a cyst, facilitation of orthognathic surgery, orthodontic abnormalities, and pain. There were 11 males and 14 females between 17 and 32 years of age with a mean age of 24 years. None of the patients had significant medical history or any complaint of pericoronitis in the preceding two weeks of the surgery. The details of the study were explained to the patients and informed consent was obtained. All surgical procedures were performed by two surgeons who had equal academic degrees. The two lower third molar teeth were removed in different sessions leaving 15 days between the two surgical procedures. The patients were assigned to test and control groups by random selection. The one side served as control and on the other side local haemostasis was achieved (test). Regional mandibular anaesthesia was administered in the same way on each occasion using articaine with $0.006 \mathrm{mg} \mathrm{ml}^{-1}$ adrenaline. In both groups, a buccal flap was reflected and a sufficient amount of bone was removed with a round bur. After the removal of the tooth, the socket was irrigated with saline. In the control group, the wound was primarily closed with silk sutures. In the test group, an absorbable haemostatic agent, oxidised regenerated cellulose, (Surgicel, Ethicon Inc. Johnson and Johnson Company, USA) was placed in the extraction cavity following gauze packing for 3 minutes to prevent bleeding. In addition, soft tissue focal bleeding points were eliminated by electrocautery and the wound was primarily closed with silk sutures after complete local haemostasis had been achieved. In the post-operative period, all the patients were prescribed the same analgesics (nimesulid). 
All the patients were examined by the same clinician pre-operatively and at 1 and 3 days post-operatively. Data was collected regarding the duration of the surgery (minutes), facial swelling $\left(\mathrm{mm}^{2}\right)$ and mouth opening $(\mathrm{mm})$.

Mouth opening, as the maximum distance between the edges of the central incisors, was measured with a Boley gauge. The measurement was repeated three times and the values were averaged.

Facial swelling was measured by a point counting technique on standard postero-anterior skull radiographs in terms of square millimetres. Two reference lines were drawn on the radiographs one of which was in the vertical and the other in the horizontal plane (Fig. 1). The horizontal reference line was drawn between the tips of the right and left mastoid bones. The outer border of the mandible and the corresponding soft tissue contour were also drawn on the radiographs. The area between these lines was calculated by using a point counting technique. These calculations were repeated three times by the same author at one week intervals and the average was used for analysis.

The duration of the operation was determined as the time from the initial mucosal incision to the termination of suturing.

The data relative to the facial swelling were analysed using a nonparametric test for related samples (Wilcoxon signed rank test). The duration of surgery and mouth opening were analysed by using Student's $t$ test for related samples.

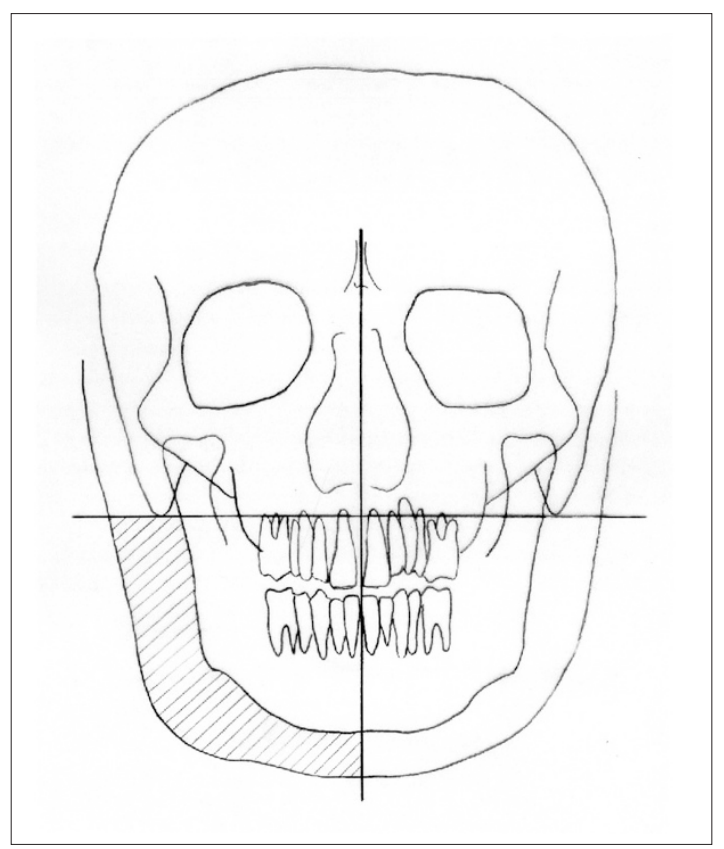

Fig. 1 Diagrammatic view of the facial swelling area

\section{RESULTS}

The mean facial swelling values for the two groups are shown in Table 1 . The majority of the patients exhibited facial swelling in varying degrees. Percentage value (Pre-op - Post-op/Pre-op $\times 100$ ) was used in this study because of the greatness of the inter-individual variation. On post-operative day 1 , the mean facial swelling was $26 \%$ in the control group, and $17 \%$ in the test group where haemostasis was achieved. On post-operative day 3, the mean facial swelling was $24 \%$ and $22 \%$ for the control and the test group, respectively. There were no statistically significant differences between the groups either on the post-operative day $1(P=0.069)$ or on the post-operative day $3(P=0.56)$ for the mean values of the facial swelling.

There were no statistically significant differences $(P>0.05)$ between the groups regarding mouth opening either on postoperative day $1(P=0.281)$ and $3(P=0.052)$ (Table 2$)$. On the first postoperative day, the mean reduction in the mouth opening was $27 \%$
Table 1 Descriptive statistics of the facial swelling for the both control and test groups. The values provided show the percentage increase of the measured area when compared with the pre-operative values

\begin{tabular}{llllll}
\hline Group & Time & N & Mean & SD & Range \\
\hline Control & Post-op 1 & 25 & 26.60 & 20.71 & $0.00-81.82$ \\
& Post-op 3 & 25 & 24.88 & 14.35 & $2.50-52.17$ \\
& & & & & \\
\hline Test & Post-op 1 & 25 & 17.73 & 15.28 & $0.00-51.16$ \\
& Post-op 3 & 25 & 22.24 & 18.82 & $1.45-61.60$
\end{tabular}

Table 2 Descriptive statistics of the ability of mouth opening for both groups

\begin{tabular}{llllll}
\hline Group & Time & N & Mean & SD & Range \\
\hline Control & Post-op 1 & 25 & 27.04 & 16.50 & $1.89-57.78$ \\
& Post-op 3 & 25 & 28.17 & 16.41 & $0.00-67.92$ \\
& & & & & \\
\hline Test & Post-op 1 & 25 & 23.77 & 16.61 & $0.00-51.79$ \\
& Post-op 3 & 25 & 22.22 & 11.35 & $0.00-41.67$
\end{tabular}

Table 3 Comparison of the operation times for the groups

\begin{tabular}{lccccc}
\hline Group & $N$ & Mean & SD & Range & Pvalue \\
\hline Control & 25 & 6.22 & 2.64 & $3-15$ & $<0.001$ \\
\hline Test & 25 & 8.86 & 2.45 & $6.5-15$ & $<0.001$
\end{tabular}

in the control group and 23\% in the test group. On the third postoperative day, the mean values were $28 \%$ and $22 \%$ in the control and test groups, respectively.

There was a statistically significant difference $(P<0.001)$ between the mean operation time for the test and control groups (Table 3). The mean duration of operation for the test group was longer because of the extra time needed to obtain local haemostasis.

\section{DISCUSSION}

In some patients following impacted third molar surgery, facial swelling is accompanied by a purplish ecchymosis and/or haematoma. This condition raises the question whether bleeding has any effects on facial swelling. If this were indeed the situation, the vast majority of studies previously carried out on the minimisation of oedema would have to be re-examined, indeed a few studies related to this issue have been performed for this purpose. The study by Dubais et al. ${ }^{5}$ showed that primary wound closure led to more post-operative problems than secondary wound closure after third molar surgery. It is now generally accepted that a mandibular third molar socket should be left partially open to facilitate post-operative irrigation and to decrease post-operative morbidity. This procedure has been called secondary closure. The different authors achieved this goal by making a small window in the flap before closure or by inserting a foreign body in the socket such as medicamented cones or drains. ${ }^{7}$ Rakpirasitkul and Pairuchvej's ${ }^{6}$ study revealed that the post-operative oedema and the degree of bleeding was less in the primary closure and tube drain group than in the no drain group. In another study, Forsgren et al. ${ }^{1}$ indicated that application of cold dressing does not influence the post-operative oedema. Roynestal et al. ${ }^{8}$ have also stated that soft laser treatment has no beneficial effect on swelling after third molar surgery. To the best of our knowledge, there is no study to date on the results of the practice of local haemostasis after the extraction of impacted mandibular third molars. In the present study, the effects of a haemostatic agent on facial swelling following third molar removal were compared with those of simple primarily wound closure. 
It is quite difficult to quantify swelling in the maxillofacial region because of its topography. A number of different techniques have been used to measure post-operative swelling. MRI or stereophotogrammetry which give measurements in threedimension are the two that are more advantageous. ${ }^{9,10}$ However, these methods may also have some disadvantages such as a high cost, claustrophobic properties, the need for complex machinery, and loss of time etc. Therefore, they are not included in routine practice. Some automatic machines or software can also measure the contours of the object to obtain the surface area. However, several studies have shown that a point counting technique represents a more reliable and efficient approach than planimetric techniques. ${ }^{11,12}$ The point counting grid, which has some point sets at distinct densities on a transparent sheet, can be used to estimate the surface area. ${ }^{11}$ In the present study, the facial swelling was measured on the posteroanterior skull radiographs by using this technique. Although this is not a new method, the use of a point counting technique to measure the area drawn on postero-anterior skull radiographs in order to evaluate facial swelling is new. Minimal post-operative irradiation and inability of performing a volumetric measurement are disadvantages of this method.

Although there was no statistically significant difference between the test and control groups, in the test group the degree of facial swelling was less than the control group at the first and third post-operative days (Table 1).

Although Surgicel is an excellent haemostatic agent for use in maxillofacial surgery, it may also cause delayed wound healing and foreign body reactions. There are few studies in the literature that have investigated post-operative bleeding after impacted third molar surgery. One of these by Senghore and Horris ${ }^{13}$ showed that a single intravenous pre-operative dose of tranexamic acid was effective in preventing excessive post-operative bleeding in patients undergoing third molar extraction, allowing uneventful discharge from hospital. However, they did not investigate the relation between post-operative bleeding and facial swelling. A local haemostatic agent and systemic tranexamic acid might be more effective in reducing post-operative bleeding and oedema when used together. This subject is currently under investigation at the authors' clinic.

In this study, it was planned to minimise the bleeding intraoperatively by using a haemostatic agent while in some studies post-operative bleeding and exudate were drained after surgery. In this study, it was thought that the facial swelling was due more to the trauma and inflammation than to the post-operative bleeding because there was no statistically significant difference between the groups.

No difference was observed between the control and test groups in their ability to open their mouths post-operatively. This suggests that the inflammatory influence on the masticatory muscles was similar whether or not a local haemostasis was applied intra-operatively.

The results of this study indicate that active measures to obtain local haemostasis after removal of impacted mandibular third molars does not save the patient from post-operative facial swelling.

1. Forsgren $H_{1}$ Heimdahl $A$, Johanson $B$, Krekmanov L. Effect of application of cold dressing on the postoperative course in oral surgery. Int J Oral Surg 1985; 14: 223-228.

2. Beirne $O R$, Hollander $B$. The effect of methlyprednisolone on pain, trismus, and swelling after removal of third molars. Oral Surg Oral Med Oral Pathol 1986; 61: 134-138.

3. Mosgau SS, Schmelzeisen R, Frölich J C, Schmele H. Use of ibuprofen and methlyprednisolone for the prevention of pain and swelling after removal of impacted third molars. J Oral Maxillofac Surg 1995; 53: 2-7.

4. Jackson D L. Determination of the anti-inflammatory effects of methylprednisolone on the sequelae of third molar surgery (disscussion). J Oral Maxillofac Surg 1999; 57: 1206-1208.

5. Dubois D D, Pizer M E, Chinnis R J. Comparison of primary and secondary closure techniques after removal of impacted mandibular third molars. J Oral Maxillofac Surg 1982; 40: 631-634.

6. Rakprasitkul S, Pairuchvej V. Mandibular third molar surgery with primary closure and tube drain. Int J Oral Maxillofac Surg 1997: 26: 187-190.

7. Brabander E C, Cattaneo G. The effect of surgical drain together with a secondary closure technique on postoperative trismus, swelling and pain after mandibular third molar surgery. Int J Oral Maxillofac Surg 1988; 17: 119-121.

8. Roynesdal A K, Bjornland T, Barkvoll P, Haanaes H R. The effect of soft-laser application on postoperative pain and swelling. A double-blind, crossover study. Int J Oral Maxillofac Surg 1993; 22: 242-245.

9. Llewelyn J, Ryan M, Santosh $C$. The use of magnetic resonance imaging to assess swelling after the removal of third molar teeth. Br J Oral Maxillofac Surg 1996; 34: 419-423.

10. Mocan A, Kisnisci R, Ucok C. Stereophotogrammetric and clinical evaluation of morbidity after removal of lower third molars by two different surgical techniques. J Oral Maxillofac Surg 1996; 54: 171-175.

11. Sahin B, Emirzeoglu M, Uzun A, Incesu L, Bek Y, Bilgic S, Kaplan S. Unbiased estimation of the liver volume by the Cavalieri principle using magnetic resonance images. EurJRadio/2003; 47:147-170.

12. Jorgen $H$, Gundersen $G$, Boysen $M$, Reith A. Comparison of semiautomatic digitizertablet and simple point counting performance in morphometry. Virchows Arch B Cell Pathol Incl Mol Pathol 1981; 37: 317-325.

13. Senghore $N$, Harris M. The effect of tranexamic acid (cyclokapron) on blood loss after third molar extraction under a day case general anaesthetic. Br Dent J 1999; 26: 634-636. 